\title{
Atención médica y diferencia cultural. Acceso a atención médica de mujeres musulmanas en Bogotá*
}

\section{Medical Care and Cultural Difference. Access to Medical Care by Muslim Women in Bogota}

\section{Atendimento médico e diferença cultural. Acesso a atendimento médico de mulheres muçulmanas em Bogotá}

Fecha de recepción: 24-05-16 Fecha de aceptación: 18-08-16 Disponible en línea: 30-11-16 doi:10.11144/Javeriana.rgyps15-31.amdc

Cómo citar este artículo:

Arboleda-Sarmiento JC, Suárez-Montañez R. Atención médica y diferencia cultural. Acceso a atención médica de mujeres musulmanas en Bogotá. Rev. Gerenc. Polít. Salud. 2016; 15(31): 30-49. http://dx.doi. org/10.11144/Javeriana.rgyps15-31.amdc

Juan Camilo Arboleda-Sarmiento**

Roberto Suárez-Montañez***

* $\quad$ Este documento es producto de la investigación realizado durante el año 2015 para el trabajo de grado de la Maestría en Salud Pública de la Pontificia Universidad Javeriana, Bogotá, Colombia.

** Investigador principal, antropólogo, MA. Líder poblacional, Subsecretaría de Salud Pública, Secretaría Distrital de Salud, Bogotá, Colombia. Dirección: Grupo Antropología Médica, Departamento de Antropología, Facultad de Ciencias Sociales, Universidad de los Andes. Carrera 1 No. 18á-10. Bogotá D. C. Correo electrónico: arboleda.j@

javeriana.edu.co

*** Tutor del trabajo de grado. Antropólogo, profesor asociado del Departamento de Antropología de la Universidad de Los Andes, Bogotá, Colombia. Correo electrónico: rsuarez@uniandes.edu.co 


\section{Resumen}

Desde 1991 Colombia se define constitucionalmente como un país multicultural. Sin embargo, la aplicación de esta definición es aún incierta. Sobre esta base, el texto explora la relación entre diversidad cultural y acceso a servicios de salud, a partir del caso de las mujeres musulmanas en la ciudad de Bogotá, Colombia. Se plantea cómo los estilos de vida, el género y los sistemas de creencias afectan las trayectorias de cuidado y el encuentro terapéutico de personas definidas como musulmanes en los servicios de atención médica. Se desarrolló un trabajo de corte cualitativo, donde se articulan la salud pública y la antropología médica. Se encontró que el acceso a la atención médica de las mujeres musulmanas está mediado por interacciones sociales que facilitan los desencuentros médicos. De allí que la promoción de la salud y la prevención de la enfermedad requieran la comprensión e integración de la cultura como categoría mediadora en la salud pública.

Palabras clave: multiculturalismo; islam; género; trayectorias terapéuticas; acceso a atención médica

\section{Abstract}

Since 1991, Colombia has been defined as a multicultural country. However, the application of this definition is uncertain still. With this background, our text explores the relationship between cultural diversity and the access to health services based on the case of Muslim women in Bogota, Colombia. We propose how lifestyles, gender, and belief systems affect the path of care and the therapeutic meeting of people defined as Muslim in the health care services. We developed a qualitative work articulating public health and medical anthropology. We found that the access to medical care of Muslim women is mediated by social interactions facilitating medical disagreements. From there we surmise that health promotion and disease prevention require the understanding and integration of culture as a mediating category in public health.

Keywords: multiculturalism; Islam; gender; therapeutic paths; access to health care

\section{Resumo}

Desde 1991 Colômbia é definida constitucionalmente como país multicultural. No entanto, a aplicação desta definição ainda tem incerteza. Sobre esta base, o texto explora a relação entre diversidade cultural e acesso a serviços de saúde, a partir do caso de mulheres muçulmanas na cidade de Bogotá, Colômbia. Coloca-se como os estilos de vida, o gênero e os sistemas de crenças afetam as trajetórias de cuidado e o encontro terapêutico de pessoas definidas como muçulmanos nos serviços de atenção médica. Um trabalho de tipo qualitativo onde é articulada a saúde pública e a antropologia médica foi desenvolvido. Encontrou-se que o acesso ao atendimento médico das mulheres muçulmanas está mediado por interações sociais que facilitam os desencontros médicos. Daí que a promoção da saúde e a prevenção de doença requeiram a compreensão e integração da cultura como categoria mediadora na saúde pública.

Palavras-chave: multiculturalismo; islame; gênero; trajetórias terapêuticas; acesso a atendimento médico 


\section{Introducción}

A partir de la Constitución de 1991, Colombia se definió como país multiétnico y multicultural (Art. 7), poblado y divido en grupos sociales que debían ser reconocidos en su diferencia. El principio de la heterogeneidad poblacional colombiana se pensó como un concepto donde la diversidad étnica ha sido una categoría que prima en términos de legitimidad social, más que la diferencia cultural. Por ello, los otros diferentes de la nación se han constituido dentro del imaginario como una unidad demográfica definida por su perfil étnico y su ubicación en territorios alejados de los centros urbanos.

En este sentido, lo étnico es un término polisémico que usualmente se define a partir de conceptos geopolíticos, culturales o eugenésicos. Además, es usado para narrar diferencias grupales o construir diferencias identitarias. Por ello, muchos estudios sobre prácticas en salud y el encuentro de diferentes culturas médicas, sistemas diagnósticos y modelos explicativos de la salud y la enfermedad se fundamentan en representaciones estereotipadas, las cuales reducen los grupos humanos a una sola condición que, en todos los casos, plantea una racialización de la diferencia a partir de rasgos fenotípicos o experiencias sociales compartidas transgeneracionalmente (1-4).

En muchas definiciones de etnia, es posible observar cómo esta categoría se convierte en una término funcional y legitimador de condiciones regionales o nacionales de inclusión o exclusión social de los ciudadanos en el Estado-nación. En el caso colombiano, la íntima asociación entre etnia y raza plantea grandes cuestionamientos sobre cómo es pensada la diversidad cultural y cómo se ubican aquellos grupos indígenas, afrocolombianos, negros, raizales, palenqueros y gitanos (5). Es allí donde la diferencia y la respuesta social a esta se hacen difusas, más aún cuando estos individuos no pueden ser racializados por su fenotipo y porque, además, viven una cotidianidad urbana en la cual se diluyen en la masa poblacional del país (6).

En el campo de la salud pública y del acceso a servicios de salud oficiales, este hecho plantea un reto importante, ya que analizar cómo la diferencia cultural es imaginada, cómo opera y cómo se construye y funcionaliza dentro del sistema de salud, implica reflexionar tanto sobre las trayectorias burocráticas como sobre la cultura para entender el encuentro terapéutico entre un usuario, representado y esencializado a partir de su condición de un otro diferente, y el sistema oficial de salud (7). En otras palabras, planteamos analizar cómo las prácticas en atención y cuidado de la salud entre individuos culturalmente diferentes y los servicios de salud oficiales se construyen o deconstruyen dependiendo de cómo los imaginarios sobre la otredad se escenifican en la relación y las racionalidades entre proveedor en salud y usuarios del sistema. Este es el caso de la comunidad musulmana (8), buscamos "comprender la racionalidad de las acciones desarrolladas por los sujetos y grupos sociales". La comunidad musulmana residente en Bogotá se estima en alrededor de 1500 personas (9).

Los musulmanes históricamente han sido una población objeto de exclusión social en el mundo cristiano. Se les ha estereotipado como infieles, enemigos de la civilización, ciudadanos anómalos, miembros de una civilización imperfecta. Han sido objeto de pogromos u obligados al exilio, entre muchas otras prácticas (10). Estas imágenes del árabe y del mundo social y cultural que lo rodea desde el 9/11 vuelven a reforzarse, bien sea por sus planteamientos sobre el género femenino, o por sus planteamientos políticos en contra de la democracia occidental. La figura del árabe es entonces asociada al musulmán y 
se lo representa a partir del suicida-terrorista y de la mujer arropada con un tipo de vestido que oculta su cuerpo de la mirada de los demás, hecho que no permite ningún tipo de juicio de valor y donde la intimidad del cuerpo de la mujer musulmana permanece intacta, aun en los espacios públicos.

Los dos ejemplos mencionados justificaron tanto el estudio realizado como el análisis presentado en este documento. Por ello, los datos que se muestran dan cuenta del musulmán pensado como un otro, no étnico, pero sí religioso, que confronta los valores de una cultura pensada como global y escenificada como libertaria, autónoma, igualitaria y democrática. Aunque en Colombia lo étnico se reduce a minorías como los indígenas, las negritudes y el pueblo Rom, en otros contextos, árabes, chinos, caribeños, pakistaníes, indios, británicos asiáticos, británicos negros, hispanos, irlandeses estadounidenses, hawaianos, nativos americanos y créoles, entre muchos otros, son definidos como grupos étnicos.

Sobre esa base, este documento tiene como objetivo analizar el encuentro cultural entre un grupo de usuarios musulmanes y los prestadores de servicios oficiales de salud, a través de tres casos que ilustran las trayectorias terapéuticas de estas personas dentro del Sistema General de Seguridad Social en Salud (SGSSS). A este efecto, el estudio se puede ubicar dentro un marco referencial que articula conceptos de base de la antropología médica con una perspectiva de salud pública, para poder entender la vida cotidiana de una persona musulmana que busca acceder a la atención médica.

La construcción del otro musulmán: entre multiculturalismo y etnicidad

Pensar a los otros, lejanos, desde los tiempos de Herodoto (425 a. C.), ha sido fundamental en la sociedad, para dominarlos, integrarlos o simplemente intervenirlos. La imagen del otro oriental creada por los británicos y los franceses, a partir de la intención de dominación, sirvió para crear imágenes llenas de prejuicios al describir la personalidad de los orientales a quienes se quería someter (11). La vieja discusión sobre quién es el buen musulmán y quién el malo influye en nuestros propios imaginarios sobre lo que es un buen ciudadano en un contexto democrático. En los Estados Unidos, el musulmán contemporáneo es percibido como violento, causa temor entre los ciudadanos, altera el orden de una nación civilizada y, por ende, ese es el musulmán malo.

En contraste, el buen musulmán es aquel que no da miedo, es cumplidor de los preceptos religiosos y no se hace visible en el marco de la sociedad, ya que se viste, come, estudia, trabaja y lleva una vida social y cultural como cualquier otro ciudadano. Esta dicotomía, renovada a partir del 9/11, encarna las ideas políticas de las reformas migratorias de Ronald Reagan, las cuales ubicaban los valores anglosajones cristianos en un estatus hegemónico (12). Este tipo de aproximación se plantea con las poblaciones negras, para las cuales la integración y la movilidad social se logran a través de la única posibilidad de transformación o control cultural, mediante la integración profunda y natural del comportamiento civilizado, como puede ser la cultura francesa $(13,14)$.

El problema de la diferencia social, definida bien sea como étnica, racial o cultural, al ser ubicado dentro del campo de la salud permite comprender cómo se han dado los procesos de salud-enfermedad, cuidado y atención en salud desde las primeras décadas del siglo XX en las diferentes poblaciones de los Estados nación. Si bien en las últimas décadas estas inquietudes sobre las prácticas culturales en salud se han trasladado a la reflexión sobre condiciones de vida, culturas 
médicas en poblaciones que tradicionalmente no accedían a los sistemas oficiales de salud, hoy al ser usuarios corrientes del sistema, producto de su condición de ciudadanía, la cual les permite mantener su identidad cultural y acceder al servicio, es posible vislumbrar la complejidad entre el problema de la universalidad y el relativismo cultural dentro del Sistema General de Seguridad Social.

El caso de las parteras en Guatemala (15) ilustra cómo cohabitan los sistemas curativos, el sistema médico y el sistema cultural, donde la parturienta toma decisiones que considera harán más fácil su alumbramiento, y desmiente la idea romántica de la partería europea como respuesta desde la feminidad a la obstetricia, para plantear la necesidad de integrar el contexto cultural y social nativo para dar a luz. Otros autores (16) muestran, de igual manera, que la convivencia entre el sistema curativo de la medicina alópata y los saberes comunes no científicos permite la construcción de mitos, acciones curativas y creencias, incorporando el lenguaje científico propio de la medicina occidental. La discapacidad plantea un debate sobre lo que se considera normalidad biológica y aquellos grupos sociales que si bien no hacen parte de la categoría de etnia, plantean la complejidad de las diferencias culturales dentro de los paisajes sociales actuales, para así entender las necesidades y las prácticas de un grupo que se define por la diferencia biológica (17).

La diversidad cultural es un hecho central en las naciones contemporáneas. Sin embargo, al abordar las prácticas sociales que se constituyen en barreras culturales para el acceso a la atención médica, es necesario entender la relación entre cuerpo privado y cuerpo público en el marco del encuentro médico, para mirar de manera global todos los aspectos que entran en juego en el momento de la realización de la citología. Es decir, que el cuerpo femenino que se expone y se desnuda en el ámbito médico, es parte de un acto naturalizado para la medicina, pero desde la persona puede representar una ruptura con la vida íntima individual y la intimidad de la pareja.

En las guías de atención para pacientes "culturalmente diferentes" se abordan los sistemas de creencias y marcos espirituales que cohabitan en los diferentes países. El caso canadiense es significativo, ya que allí no se reconoce solamente la diversidad cultural, sino también la diversidad étnica, en la cual se busca que el cuidado en salud sea prestado dentro de un contexto social y culturalmente pertinente que ofrezca un acceso real a la atención y el cuidado médico, de acuerdo con parámetros de multiculturalidad del Estado (18,19).

Las investigaciones sobre los musulmanes han girado en torno a otras religiones monoteístas que tienen prácticas similares. En concreto, se habla de ellos junto con judíos y testigos de Jehová, además de otros grupos de fieles que hacen parte de los cismas cristianos. En estos sistemas de creencias, la normatividad de comportamientos tiene una fuerte asociación con la religión y la religiosidad de las personas, para seguir prácticas preventivas que les permitan a los individuos afrontar y resignificar la enfermedad y los síntomas, así como pensar la salud (20-23).

\section{Consideraciones metodológicas}

\section{Tipo de estudio}

Este es un estudio de corte cualitativo en salud pública, donde se incluyen herramientas de la antropología médica. Con ello se busca comprender el lugar del individuo, los grupos humanos, la cultura y lo social dentro de la complejidad del campo de la salud y la enfermedad $(16,24)$. En el caso de Colombia, 
es importante entender los procesos sociales de la atención en salud que tienen lugar a través del sistema de salud en cada región del país que interesa al Gobierno Nacional, mediante la Política de Atención Integral en Salud (PAIS).

\section{Fuentes de información}

Para esta investigación se realizó, en primer lugar, una encuesta de tipo censal, a la que respondieron 33 personas, con lo cual se creó un estudio de caso. A través de esta encuesta se entrevistó a hombres y mujeres solteras conversas al islam, en virtud de que estas últimas no tienen vínculo de parentesco con hombres que las incluyan en la información que darían sobre sus familias. Con esta encuesta se buscó caracterizar a la comunidad musulmana que vive en Bogotá en relación con los datos que aporta el Departamento Administrativo Nacional de Estadística (DANE) en el último censo poblacional. Las encuestas realizadas contenían trece preguntas, de las cuales se enuncian las más importantes: estado civil, edad, nivel de estudios, nivel de ingreso mensual (en salarios mínimos mensuales legales vigentes [SMMLV]), tipo de vivienda en la que vive, afiliación a Empresa Promotora de Salud (EPS) y a fondo de pensiones, convertido o nacido en el islam y filiación religiosa a alguna corriente de dicha religión.

Población: el musulmán local. ¿Del turco comerciante al terrorista saudí?

La población árabe musulmana a lo largo de su historia ha sido llamada de diferentes maneras: mahometanos, infieles, moros, entre otras. En Colombia, la forma usual de denominarlos fue a través de la palabra turco. Este término se usaba para referirse a aquellas personas que eran comerciantes puerta a puerta, pero que por su lenguaje eran asimilados a un grupo humano que venía del Medio Oriente, aunque en términos reales eran migrantes originarios del antiguo Imperio Otomano, desintegrado al término de la Primera Guerra Mundial.

El turco, como figura del comerciante, se integró en el folclor costeño y cundiboyacense de la sociedad colombiana como aquel individuo extraño en su lenguaje y en sus costumbres. Comía, hablaba, se vestía, rezaba y pensaba de manera inusual —así como Heródoto hablaba de los bárbaros por su balbuceo lingüístico ininteligible-, con el acento gutural que describe Sánchez Juliao cuando caricaturiza la manera en que hablaba Afife Matuk en el cuento "Abraham al humor" (25), incluido en la obra Una Década David Sanchez Juliao 1973-1983 y que Cunninghame ilustra en Cartagena y las riberas del Sinú. En Bogotá el inmigrante turco (26-28) se encontraba en la así llamada "calle de los místeres”. Según recuerdan algunas personas mayores, sus ventas estaban ubicadas en la Carrera $7^{\mathrm{a}}$ con Calle 13, donde "los místeres". Eran hombres con bigotes negros poblados y enroscados en los pómulos que atendían sus negocios. Esta migración, en una importante proporción de cristianos menonitas, se asentó en la Costa Atlántica, tal como lo anota Sánchez Juliao cuando se refiere a la Lorica saudita en el cuento "El flecha" (26).

El islam, tal como un entrevistado afirmó, hace parte de la nación colombiana desde hace mucho tiempo, incluso antes de la caída del Imperio Otomano. Para él, este hecho se puede observar en la letra de la canción " $\mathrm{La}$ rebelión”, de Joe Arroyo: "Cuando aquí llegaban, esos negreros africanos en cadenas besaban mi tierra". Solo los musulmanes besan la tierra cuando se postran para adorar a Alá.

Durante la década de los setenta la comunidad musulmana dejó de estar en secreto en las inmediaciones de la plaza de Bolívar (9), 
y en 1978 se fundó una sala de reunión para orar, una musalla, con base en la población de palestinos, sirios, pakistaníes y uno que otro colombiano convertido al islam. En los últimos años, la comunidad que se reunía en la musalla del centro, a escasas cuadras de la Carrera 10å , creó nuevos espacios de oración. En el 2007 la mezquita Estambul, en el 2010, el centro de estudios Al Qurtubi, y en el año 2013 se construyó la mezquita de Bogotá Abu Bark.

Los musulmanes no tienen un barrio, como el barrio judío que se configuró en los alrededores de la Calle 94 -Avenida Estado de Israel-, donde se asentaron muchos judíos ashkenazim, principalmente polacos, o cerca del Centro Andino, donde viven algunos judíos sefarditas de Bogotá. Hoy, la población musulmana de la ciudad no tiene una localidad privilegiada. Por el contrario, se ha ubicado en las diferentes zonas que conforman la urbe.

De acuerdo con las entrevistas realizadas a los líderes de las mezquitas, y la documentación de los feligreses de la mezquita $\mathrm{Al}$ Qurtubi que respondieron la encuesta, los musulmanes de Bogotá son colombianos en su mayoría y provienen de las diferentes regiones del país. Corresponden al $73 \%$ de los encuestados, contra el $27 \%$ de extranjeros que siguen siendo la base de la comunidad, contrariamente al estereotipo de musulmán local que es asociado a la Costa Caribe.

La población musulmana proviene de centros urbanos importantes como Bogotá, Villavicencio, Cali y Popayán, y ha tenido presencia en el país al menos por tres generaciones. Son hombres y mujeres de diferentes grupos etarios que siguen un estilo de vida dentro del cual prácticas devocionales, pautas alimentarias, formas de interacción social, indumentarias tales como el uso de hiyab y la hata les permiten mantener y reproducir su identidad social. De los encuestados, el $76 \%$ estaba afiliado al SGSSS. Eran personas con educación superior, de ingreso medio, pertenecientes a estratos socioeconómicos entre el 3 y el 6.

\section{Instrumentos y estrategias de recolección}

El estudio se planteó a partir de dos casos que ilustran la relación entre cultura, sociedad y alteridad en el acceso a atención médica en una población musulmana: atención obstétrica y ginecológica y del cáncer de mama. El trabajo de campo se desarrolló con los feligreses que asisten a una mezquita en Bogotá, y varias entrevistas tuvieron lugar en espacios frecuentados por los miembros de la comunidad musulmana en el centro de Bogotá. Así mismo, se respetaron las normas de interacción social de género y de anonimato de la población. Por ello, algunas entrevistas con mujeres fueron vía telefónica y otras en presencia de testigos que dieron cuenta de que se mantuviera el orden establecido por sus costumbres.

Entrevistas semiestructuradas. Este es un método que, a través de una guía, un cuestionario con preguntas abiertas, busca obtener la información relevante para el estudio (27). Se realizaron nueve $(n=9)$ entrevistas, de las cuales se grabaron siete $(n=7)$. De estas, siete se hicieron en las sedes de las mezquitas y dos mediante correo electrónico. Todas las personas participaron voluntariamente en el estudio, eran mayores de edad, seis conversos, y hacían parte de la comunidad desde hacía mínimo tres años. En la investigación no se incluyeron los nombres reales de las mujeres entrevistadas. El cuestionario constaba de cinco preguntas, en las cuales se incluyeron ocho categorías analíticas del trabajo: ubicación social, conversión, organización social y comunidad, género, cultura médica, itinerarios curativos y migración. 
Etnografía. La etnografía como técnica de testimonio visual buscó, por una parte, describir prácticas, y por otra, entender desde la perspectiva del individuo las racionalidades que se encuentran detrás de cada acción terapéutica (27). Se realizó en las mezquitas y en los centros de salud, en dos momentos de la atención, uno previo a la consulta y el otro después de esta. La primera fase se llevó a cabo de octubre a diciembre del 2014, en tanto que la segunda se realizó entre febrero y julio del 2015.

\section{Criterios de análisis y tipo de análisis}

El fundamento analítico de este estudio buscó articular el problema de la cultura a los determinantes sociales de la salud, y con ello entender cómo se tejen las trayectorias en salud, cómo se significan los encuentros terapéuticos y cómo se vive la atención en salud por parte de las personas musulmanas. Esto con la finalidad de ubicar la relación cultura-salud en un lugar privilegiado, como epicentro de las relaciones sociales, especialmente de aquellas que conciernen al contínuum salud-enfermedad y trayectorias de atención en salud.

\section{Marco analítico}

El análisis se construyó, fundamentalmente, a partir de dos ejes. El primero, mediante un análisis de contenido que buscó entender cómo se construyen las narrativas escritas, orales y gestuales para analizar el contexto en el cual tienen lugar los encuentros terapéuticos (29). En segundo lugar, el marco interpretativo de los datos establece una cercanía con la teoría fundamentada, en donde el dato obtenido en el trabajo de campo se articuló y dialogó con fuentes teóricas desde la sociología y la antropología médica, con las fuentes secundarias del islam. Es decir, que el análisis conecta las relaciones entre los significados de las experiencias en salud, las percepciones de los individuos y sus prácticas en la vida cotidiana.

\section{Hallazgos}

Ir al médico es una práctica social naturalizada en el mundo contemporáneo. Asistir a una consulta médica, usualmente implica unos preparativos burocráticos y emocionales que son ajustados a unas convicciones y a un consenso social sobre la relación entre la persona, el paciente y el proveedor en salud: el médico. La consonancia o disonancia entre ambos individuos se escenifica por las preferencias propias de cada paciente y los acuerdos normalizados sobre lo que son los roles de cada uno y la forma de interacción en el espacio de la consulta médica. Sin embargo, este encuentro deja su naturalidad en el momento en que intervienen factores como el estilo vida, la religión, las ideologías políticas, las relaciones de clase social, el género o el racismo (28). En el caso de los musulmanes, el encuentro médico está mediado por el islam, religión que define la trayectoria en salud de sus practicantes y las formas de hacer uso del SGSSS.

La relación religión, cuidado de la salud, SGSSS, aspectos del cuerpo que sufre y la persona enferma que busca respuestas a malestares sentidos, pone de manifiesto las tensiones entre el sistema de creencias y el sistema de cuidado oficial. En este sentido, las prácticas corrientes y vividas como normales en los encuentros terapéuticos son cuestionados por otras culturas. Así, desde otra perspectiva, el cuerpo expuesto, tocado, auscultado, desnudo dentro del espacio de la consulta médica implica una pérdida de intimidad, una consecuencia moral, psicológica, emocional y religiosa que debe abordarse bajo unos parámetros definidos por un sistema de creencia particular que permite el afronta- 
miento del encuentro médico-paciente. En el marco del islam, el cuerpo no es solo una unidad anatómica y clínica, es un cuerpo social, político y religioso que encarna una forma de ser y estar en el mundo, de acuerdo con unos principios religiosos (30).

En el islam el cuerpo es un espacio íntimo que solo puede ser observado por una persona del mismo sexo; es decir, que un hombre debe ser atendido por otro hombre y una mujer debe ser atendida por otra mujer. Solo en casos extremos se rompe esta regla de la Sunna, y es debido a la carencia de opciones de atención médica. Por ello, la experiencia de la salud, la enfermedad, las trayectorias en salud, el acceso al cuidado de la salud y las rutas terapéuticas del musulmán sonfundamentalmente una experiencia social y cultural. Si bien los medicamentos, los exámenes diagnósticos y las terapias físicas no son dejados de lado, ni son negados, la adherencia a ellos está determinada por la relación religión, cultura y salud.

Lo anterior se puede observar en el ejemplo de los ayunos rituales durante el mes del Ramadán. En ese momento en la vida espiritual del musulmán, la persona se ve obligada a modificar horarios para cumplir con las guías clínicas sobre la ingesta de medicamentos, así como con la observancia religiosa. Por ello, los eventos burocráticos comunes en la vida de los afiliados al SGSSS, tales como llamar a un call center para buscar una cita médica en una IPS, así como su ritualidad religiosa diaria, conforman una unidad de sentido que les permite vivir bien su condición de ser musulmanes en la sociedad.

\section{Atención en ginecología: la intimidad expuesta}

Al observar las cifras de ocurrencia de cáncer de cuello uterino, que lo ubican como el cuarto más frecuente en mujeres, el hecho de que haya más de 270000 muertes por esta causa, y que de estas cifras el $85 \%$ corresponda a los países menos desarrollados, se puede comprender por qué se han desarrollado protocolos, guías e intervenciones en salud pública para su control (31). Según el Instituto Nacional de Cancerología (INC) (32), en Colombia la incidencia de cáncer de cuello uterino es de 8.2 muertes por cada 100 000, lo que representan 19861 casos. Pese a ello, esta enfermedad es un problema de salud pública, ya que continúa siendo la segunda causa de muerte en las mujeres de América Latina.

En este sentido, el acceso a servicios de ginecología y obstetricia se ha convertido en un eje importante para su detección temprana. Por ello, en el ámbito nacional, el Ministerio de Salud y el INC han promovido intervenciones, monitoreo y cobertura para el diagnóstico y tratamiento temprano del cáncer cervicouterino (33). Así mismo, el examen de papanicolaou - la citología cervical- para la detección de este cáncer se presenta socialmente como un procedimiento necesario para la prevención y detección temprana, disminuir la morbimortalidad y promover el bienestar de las mujeres.

Sin embargo, la dificultad para acceder a servicios médicos por parte de poblaciones definidas por su diferencia cultural, es importante y conlleva importantes consecuencias en términos de inequidad y discriminación social $(28,34)$. De una parte, aspectos tales como el vestuario, el lenguaje verbal y no verbal, las creencias y los tabúes corporales, entre muchos otros, pueden ser percibidos o significados como barreras sociales y culturales que entorpecen la comunicación e interacción entre el médico, la enfermera, el personal de apoyo burocrático y el paciente. Pero, por otra parte, la gran importancia de los valores científicos y profesionales de la medicina exige de la persona un acto de sumisión cultural a estos. De esta forma, el 
espacio de la consulta es imaginado como un lugar donde la ciencia prima sobre cualquier otro sistema. En otras palabras, lo individual y lo cultural, en cuanto que aspectos que marcan la experiencia de la enfermedad de la persona, pasan a un segundo plano cuando la ciencia médica surge como idea totalizante explicativa de los procesos de salud-enfermedad.

En la experiencia del islam, el ser humano y su cuerpo son sagrados, solo accesibles a los ojos de la vida marital y de Dios mismo. Por esta razón, el cuerpo se proyecta como un lugar privado e íntimo cuya exposición se limita a tiempos, lugares y personas relacionadas con lo espiritual y con el grupo familiar nuclear; es decir, personas en primer grado de afinidad y primer grado de consanguinidad. El cuerpo, bajo esta mirada, es indivisible, es uno y es pensado únicamente para Dios en el plano social, individual, temporal y territorial.

Aunque en el cuerpo femenino encarna la religión, este es vivido como un cuerpo a la vez biológico, político y social, pero que es fundamentalmente un cuerpo trascendente que acontece en la cotidianidad de la fe. En esta religión la división sagrado-profano no tiene sentido, ya que hay una constante conexión entre todos los aspectos de la vida de la persona. De allí que el encuentro médico no sea ajeno a la vida religiosa, a los valores y a la guía espiritual de las personas. Al contrario, el campo de la salud y la enfermedad es un reflejo de la vida religiosa del musulmán y es un lugar donde se expresa la ideología islámica como camino de trascendencia.

Tanto la OMS como el INC han venido desarrollando y consolidando la idea de detección temprana y prevención $(32,35)$, donde se recomienda para las mujeres que el examen se haga anualmente, y en los casos donde se presente una alta vulnerabilidad y riesgo, hasta dos veces.
El régimen general de seguridad social colombiano ha buscado como premisa fundamental el acceso universal a los servicios de salud y la promoción de condiciones pertinentes para que este acceso sea efectivo en todos los niveles, y con ello controlar el riesgo, disminuir la vulnerabilidad y asegurar, finalmente, que las personas gocen equitativamente del derecho a la salud.

En el encuentro médico multicultural, la interacción entre los grupos poblaciones, la capacidad instalada del sistema para ofrecer un acceso efectivo, el acto médico per se, se convierten en un evento que posibilita o interfiere en los procesos terapéuticos. La consulta médica, en este sentido, pone de manifiesto la complejidad del encuentro cultural en las relaciones médico-paciente, en las cuales el cuerpo, la comunicación médica y los sistemas de creencias calificados como profanos o expertos intervienen para constituir una unidad práctica y simbólica que permite la consonancia o disonancia de lo que es y se espera de la atención en salud.

En el sistema de salud la forma usual de acceder al examen de papanicolaou transcurre por etapas: en primera instancia, la mujer es informada sobre las condiciones básicas para realizarlo. Ella debe haberse abstenido de tener relaciones sexuales en los tres días previos, y se le hace una serie de preguntas en las que en algunos casos se indaga por el número de parejas sexuales que ha tenido. En segundo lugar, se debe acercar al servicio de salud vistiendo ropas cómodas, y allí se le dirige al espacio indicado para el examen, donde debe descubrirse la parte inferior de su cuerpo para permitir la visibilidad del área que se va a examinar, para finalmente ubicarse en la cama ginecológica, apoyar los pies en los estribos y ser cubierta con una sábana desde la cintura hasta las piernas. Luego ingresa la persona indicada y manipula el instrumental para la toma de la muestra, y una vez tomado 
el examen le permiten a la mujer volver a vestirse. Este recorrido, que es una práctica usada naturalmente y en relación con la cual no hay ningún tipo de cuestionamiento, ya que encarna ciencia, técnica, prevención y control, dentro una lógica humanitaria, es fracturado cuando intervienen los sistemas de creencias.

Cuando las condiciones para que la mujer pueda tomarse el examen no se cumplen, o cuando ella siente que las condiciones estructurales para la toma de este violan las guías religiosas, la respuesta social en las IPS se asocia con los imaginarios de la mujer musulmana, sometida e irreflexiva con respecto a su proceso de salud-enfermedad. Por ello, cuando se presenta un acto disruptivo en la normalidad del examen, la pregunta que se plantea en los centros de salud es: ¿por qué no se hace el examen, si es por su bien, si es inofensivo y no tiene consecuencias? Esta pregunta, que desde la visión médica es comprensible, pierde su fuerza al ser pensada desde lo cultural, ya que invisibiliza las emociones y las percepciones de la mujer y reduce el examen a una simple técnica inconexa con el mundo de las personas que cosifica el cuerpo de la mujer $(30,36)$. El cuerpo de la mujer pierde allí su condición privada y se expone en la cama ginecológica bajo una condición liminal entre lo mecánico-orgánico y lo simbólico asociado al género femenino.

El examen, presentado socialmente como deseable y necesario para el bien de la mujer, se convierte en un lugar simbólico de negación cultural que causa la invisibilización de la diferencia, ya que para la mujer musulmana, descubrir el cuerpo, la exposición pública de este, es una práctica que solo transcurre en la intimidad del ser. En el Occidente contemporáneo, tanto el cuerpo como la vagina son percibidos y expuestos públicamente como lugares estéticos, eróticos, espacios de placer y procreación. En el marco del islam pueden tener el mismo rol, pero constituyen ante todo un lugar privado que se expone en el marco de la vida conyugal. Pensar la vagina solamente desde una mirada técnico-médica, o como un área que se examina asépticamente, para la mujer musulmana implica abandonar temporalmente su sistema de creencias y sustraer el cuerpo del contexto religioso, hecho que la pone en tensión con las guías de comportamiento consensuadas con la religión y el sistema de atención médica.

La mujer musulmana opta por ir vestida de acuerdo a su cultura, con su hiyab, con túnicas largas (abaya, caftan), sueltas, vestidos largos que le permitan descubrir fácilmente la vagina y mantener cubierto el resto del cuerpo (piernas, muslos, pantorrillas y tobillos), sin dar lugar a la desnudez o el descubrimiento corporal. La mujer musulmana usualmente observa que las mujeres occidentales tienen menos reparo en desvestirse de la cintura hacia abajo, porque "ir al médico" es un acto normal y, por lo tanto, lo que pasa en los espacios médicos es también normal (Entrevista a Claudia, 13/06/2015). Para Luisa, quien viajó a los Estados Unidos ya siendo musulmana, y que tuvo que acceder a los servicios de atención médica para un examen de citología, afirmó que "no sintió nada". Para ella, el examen fue muy técnico, breve, sin tiempo para sentir pena, y durante el examen no se sintió agredida, ni intimidada.

Pero no sentir nada hace parte de lo que debería ser la experiencia técnica sobre el cuerpo. La ausencia de sensaciones, no implica una emocionalidad plana, ni una técnica adecuada durante el examen; es una narración que expresa el respeto por lo íntimo y lo sagrado. Cuerpo, privacidad, sexualidad son una unidad física y simbólica que al ser articulada con otros aspectos de la vida cotidiana del musulmán constituye lo que puede ser considerado un estilo de vida, en 
el cual las normas son vividas como un acto que posibilita una vida de calidad. El examen de papanicolaou presupone la existencia de condiciones de riesgo y vulnerabilidad que permiten detección y, consecuentemente, la intervención en mujeres que han tenido una actividad sexual.

$\mathrm{Al}$ analizar el riesgo y la vulnerabilidad, para las mujeres musulmanas estos deben ser pensados en términos de la observancia de la Sunna — la tradición consuetudinaria musulmana-, que afirma que la vida sexual se inicia con la vida marital, no antes, ya que se consideraría adulterio. En las mujeres musulmanas conversas se presenta una doble condición temporal y circunstancial, su historia previa y su vida actual. El examen se inscribe en juego de tiempos donde se mira la historia de vida pasada de estas mujeres, pero ponderando su condición actual, ya que es esta la que guía su praxis social en el momento presente. Por lo tanto, el acercamiento al examen por parte de las conversas, se hace mirando el pasado, pero teniendo en cuenta que el momento actual se desarrolla dentro del marco de la observancia religiosa, y con ello se excluye la condición de vulnerabilidad y de riesgo.

La percepción del examen, pre-durante-post está enmarcada en una cultura religiosa que no se disocia de la salud tal como es propuesta por el SGSSS. Sin embargo, para la mujer musulmana los estereotipos y prejuicios por parte de los prestadores de servicios son percibidos como las principales barreras que le dificultan el acceso real a los servicios de salud (34). Por ello, el examen de papanicolaou, aunque sea clave para la prevención del cáncer, su implementación en estas poblaciones plantea un desafío cultural, tanto para la mujer musulmana que lo busca como para el personal en salud que lo practica.

\section{Embarazo y parto: más allá de} la práctica clínica

La obstetricia, como sub-área de la medicina, tiene ideas particulares sobre la reproducción, el cuerpo de la mujer y las manipulaciones para la extracción del feto. La salud sexual y reproductiva de las mujeres ha sido pensada, usualmente, por hombres, los cuales han determinado las formas pertinentes de lo que es y cómo debería ser el proceso de gestación y de parto (33). Los grandes avances en este campo, llevados a cabo por médicos famosos como Marion Sims, permiten entrever las formas sociales que hicieron posible anclar una visión de la mujer embarazada y su seguimiento médico. En la historia de la obstetricia en Occidente es evidente que esta ha sido ejercida, fundamentalmente, por hombres. Fueron ellos quienes asumieron el rol y sacaron del panorama social a las parteras tradicionales, las cuales eran los actores naturales en el parto.

La partería fue, tradicionalmente, un asunto de mujeres en donde los hombres tenían un rol ad hoc. Sin embargo, fueron hombres quienes escribieron los primeros manuales de obstetricia y quienes paulatinamente tomaron el rol de las parteras tradicionales. El parto, al dejar de ser un espacio y un evento vital atendido solo por mujeres, se convirtió en un espacio científico liderado por hombres $(37,38)$. Harding (39) plantea que la ciencia no se hace desde el punto de vista de las mujeres. La ciencia se encarnó en las posturas masculinas que consideran el punto de vista de los hombres como la perspectiva natural y general. En este sentido, la obstetricia, influida por un pensamiento masculino, se convirtió en una forma social de mantener la dominación sobre el cuerpo de las mujeres. La medicalización del cuerpo de la mujer y del proceso gestacional, es el resultado de las influencias de las diferentes fuerzas sociales (médicas, políticas, económi- 
cas y culturales) que definen y determinan la idea de la gestación y del cuidado esencial, para que el feto pensado como futuro ciudadano pueda desarrollarse y nacer en óptimas condiciones (40).

A este efecto, la trayectoria burocrática dentro del sistema exige los controles ginecobstétricos desde antes del embarazo, para garantizar que el cuerpo biológico pueda resistir el proyecto gestacional. Consultas, ingestas, ecografías, controles prenatales construyen un recorrido donde la mujer y su pareja deben dar cuenta de sus historias de salud y enfermedad, ya que el médico debe conocer los antecedentes de enfermedad, la vida sexual y el número de parejas de la madre gestante, el patrón de ciclos menstruales y los antecedentes familiares de salud en temas como la hipertensión arterial, entre otros (41). El seguimiento propio del control prenatal se hace a fin de detectar un contexto favorable para el desarrollo del feto en cuanto a lo social y lo biológico, esperando que durante este no solo no haya sintomatologías que impliquen una atención especial para la madre, como preeclampsia, sino que además se preocupa por la prevención y la funcionalidad positiva del entorno familiar y el contexto social, en términos de promoción de ambientes saludables.

Todas estas medidas están sugeridas para la madre como portadora del feto, como vehículo del proceso gestacional, y en la cual recae el peso de la transformación de la vida. Sin embargo, en este conjunto de procedimientos y consejos sobre el deber ser de la futura madre se excluyen los sistemas sociales de parentesco, los sistemas de organización familiar, los roles y las funciones dentro de los hogares. En el islam el proceso gestacional y el alumbramiento poseen una carga social alta. No solo se trata de un proceso que transcurre en el cuerpo de la mujer, sino que es un proceso social donde está incluida, de una parte, la idea de masculinidad del hombre, en cuanto guía moral y proveedor económico de la familia, y de otra parte, la religión como garante de la protección de la mujer, de su bienestar y del bienestar familiar $(42,43)$.

El parto, en el marco de lo operativo, tiene dos sujetos: la madre y el neonato. Este último, al nacer bajo la tradición musulmana, y salir del cuerpo de la mujer, plantea una metáfora de vida y de la relación con la fe que profesan sus padres. Por ello, la gestación y el alumbramiento constituyen más que un evento técnico ginecobstétrico, un hecho que reafirma la nucleación familiar dentro del islam. En la ritualización del nacimiento se plantea que sea el padre la única persona del sexo opuesto que esté presente; todas las demás personas en el parto deben ser mujeres, y su rol fundamental es el de decirle al neonato las primeras palabras que oirá: el nombre de Dios. Nadie más debe hacerlo antes que él, y debe ser lo primero que escuche, más allá del sonido contextual. Así, el parto es para el islam un asunto doméstico, casero, propio de la conformación del hogar. No un evento clínico, ni científico, sino un evento que revitaliza la comunidad con la incorporación de un nuevo miembro.

Esta forma de pensar y realizar el parto sugiere repensar las ideas feministas y de medicinas alternativas y complementarias sobre el parto humanizado. Un procedimiento pensado, sentido y escenificado en términos de contextos socioculturales, lugares y personas. El parto en casa, práctica tradicional de la sociedad colombiana antes de 1950, se dejó de lado, producto del pensamiento moderno sobre la seguridad social en salud que conllevó la obligatoriedad del parto institucionalizado, hecho que se dio a partir de la implementación del Programa de Mejoramiento Progresivo de los Servicios de Salud Pública de la OPS (44). 
El procedimiento de parto, según algunas de las guías, plantea prácticas que no son contrarias a las tradiciones musulmanas (45). Para este existe una forma de vestido que les permite a las mujeres estar cubiertas durante el nacimiento. Para ello usan un mantón ancho que les cubre desde la cabeza hasta las rodillas, y solo permite la visibilidad de la vagina durante el tiempo del parto. Para las mujeres musulmanas que estaban en embarazo durante el estudio, el acceso al servicio de atención médica en ginecobstetricia les exigió un esfuerzo emocional, moral y económico para poder cumplir con sus tradiciones religiosas. Algunas mujeres debieron autogestionar la atención médica culturalmente pertinente para su parto.

A partir de la programación de este gastaron de su propio peculio familiar, con el fin de asegurarse que todos los detalles necesarios para el alumbramiento fueran de acuerdo a lo prescrito, como, por ejemplo, que todas las personas que asistiesen al parto fueran mujeres. Otras, que por razones económicas no pudieron costear la atención, tuvieron como única opción la resignación moral y cultural para afrontar la estructura ofrecida por las EPS, que no contemplaba las particularidades culturales y se centra en las necesidades clínicas dentro de la atención médica. Elvira, una mujer de 36 años que vivió un embarazo de alto riesgo, optó por la autogestión de las condiciones administrativas que pudiera controlar:

Para evitarme dificultades, lo que he hecho es cuadrar mis citas con mujeres y eso me ha facilitado mucho el asunto. Mi relación con ellas ha sido cordial y natural. Algunas curiosas preguntan, pero otras no. La que me atiende en la actualidad es una obstetra maravillosa sin cuestionamientos o miramientos, me atiende como a cualquier paciente.
Para esta mujer la resignación tiene un rol importante:

No me gusta ser atendida por hombres, es bastante incómodo para una mujer, sin embargo, vuelve y juega la lógica de la fe. En urgencias no hay opción de elegir, solo te aferras a tu suerte. En estos casos he sido atendida por hombres y mujeres, aunque por fortuna lo que son exámenes privados he contado con mujeres, pero un par de ecografías me han sido hechas por doctores. Por fortuna, es solo un pedazo de vientre el que se ve y en general los médicos son bastante correctos y respetuosos.

Este caso no representa a la mayoría de las mujeres musulmanas, ya que muchas de ellas no tienen las opciones económicas y legales para la autogestión del embarazo. Por ello, han tenido que recurrir a acciones legales para que su derecho de profesar su fe sea tenido en cuenta, en cuanto que encarna y escenifica su diferencia, no solo religiosa, sino también cultural. Para ello han solicitado, mediante derecho de petición, la posibilidad de tener partos siguiendo a su tradición religiosa, argumentando articulados de la Constitución Política de 1991 donde se afirma la igualdad ante la ley y se garantiza la libertad de conciencia y la libertad de cultos. Pero en el artículo $6^{\circ}$ de la Ley 133 de 1994 sobre la libertad de cultos no se incluye la atención en salud como parte de lo que significa el ejercicio ciudadano fundamentado en la libertad para escoger su sistema de creencias.

Las mujeres que interpusieron acciones legales lo hicieron en contra de las EPS Famisanar y EPS Compensar. Al consultar el Manual de Autorizaciones de Famisanar (46) se observa que las autorizaciones para realizar procedimientos pueden ser para colectivos poblacionales que soliciten el mismo servicio para un procedimiento clínico: "Son las autorizaciones de un mismo servicio, para 
una misma IPS, para múltiples usuarios. Por ejemplo, la diálisis mensual para el grupo de usuarios con Insuficiencia Renal" (46, p. 15), o las autorizaciones que se relacionan con procedimientos quirúrgicos, diagnósticos o terapéuticos, dispositivos médicos o medicamentos que hagan parte del plan de beneficios del SGSS; estas solicitudes deben ser respondidas en dos días hábiles por parte del comité científico. Las condiciones culturales como las solicitadas por las mujeres musulmanas no hacen parte de las condiciones especiales para los colectivos poblacionales con necesidades diferentes que no estén relacionadas con un tratamiento clínico.

Lo usual en el sistema es que se pondere lo médico en relación con lo que estipula la Comisión de Regulación en Salud (CRES). Compensar EPS, en el documento Manual para la Atención de la Gestante, pareciera plantear la oportunidad para la consideración de una atención en relación con la coherencia cultural y religiosa del paciente en virtud de sus derechos como usuario, donde se afirma que toda mujer tiene derecho a participar en los procesos de decisión (diagnósticoterapéuticos) que la afecten tanto a ella como a su hijo (47). Los procedimientos legales seguidos por las mujeres musulmanas y los planteamientos de las EPS en relación con la diferencia ponen de manifiesto la dificultad de pensar la diversidad cultural como un derecho en la atención médica y legitiman el concepto de barrera cultural como un comodín político y económico para aquellos grupos humanos que en términos políticos son culturalmente diferentes.

\section{Atención médica en oncología: cáncer de mama}

En Colombia, según el INC, en el 2015 se presentaron 7627 casos, de los cuales murieron 2226. De acuerdo con la OPS, en el año 2012 se diagnosticaron en América 408000 casos, de los cuales fallecieron 92000 (48). Este tipo de cáncer es el más común en las mujeres que habitan en América Latina y el Caribe. Para la Agencia Internacional para la Investigación del Cáncer (IARC, en su sigla en inglés) (49), conductas de riesgo tales como el consumo de anticonceptivos orales y la dieta son solo unas de las pocas conductas sintomáticas que podrían ser de riesgo, más las que aún no se conocen. Por ello, la OMS promueve la lucha contra el cáncer de mama, en el marco de programas nacionales amplios de control del cáncer que están integrados con las enfermedades no transmisibles y otros problemas relacionados (50).

En este sentido, el tamizaje de cáncer de mama es central para la OMS, ya que incluye el examen clínico, la mamografía y el autoexamen, tres aspectos que se han constituido en unidad significativa, simbólica y práctica, tanto para la detección temprana en la salud de las mujeres, como para el control de la enfermedad. Aunque la noción de prevención y control es central en los programas de salud pública, estos conceptos parecieran generar una disonancia entre grupos sociales como los musulmanes, si se tiene en cuenta que prácticas como el consumo del alcohol, el uso de anticonceptivos orales y el sobrepeso son poco probables en una sociedad de abstemios como el islam. Esta religión controla, en virtud de las leyes consuetudinarias, la dieta, y en ella se vive dentro de un sistema de intercambio marital monógamo, de acuerdo con el cual solo se tienen relaciones sexuales en el marco de la vida conyugal, y se acepta la anticoncepción y la planificación en los casos que la tradición lo permite, de conformidad con las entrevistas realizadas.

Dentro de este contexto de salud pública, el examen clínico puede constituirse en un campo de encuentros o desencuentros entre médico y pacientes, porque es el momento en el cual la posibilidad de estar enferma 
se observa y se siente mediante el tacto del cuerpo (51). El examen clínico implica desnudez, ya que se debe descubrir un área del cuerpo de la mujer que en el mundo contemporáneo está cargada cultural y estéticamente. El pecho de la mujer es un lugar particular de su cuerpo que ha sido objeto de múltiples imaginarios, de las industrias textiles, de las industrias culturales y de la medicina científica.

Los senos tienen funciones sexuales que propician la construcción del tabú corporal hacia esta parte del cuerpo (52); no solo son recipientes llenos de leche durante el periodo de lactancia, sino que son ante todo estímulos sexuales y táctiles en su uso con fines reproductivos, que incluso adquieren tanta relevancia en la construcción de la feminidad como la pueden tener las caderas. Debido a esto, el seno es parte fundamental de la estética y la identidad femenina. Una parte del cuerpo que se expone, se sugiere o simplemente se oculta. De allí que el cáncer de mama y la mastectomía sean tan importantes para el campo de la salud. La importancia del seno para la mujer se hace evidente en algunos estudios sobre mujeres mastectomizadas, donde se muestra cómo después de cirugía hay percepciones de discapacidad, envejecimiento, de ser incompletas, de mutilación que les imposibilitaban llevar una vida familiar y sexual normal (53-56).

Estas ideas sobre el seno de la mujer llevan a cuestionar cómo en el islam se construye el encuentro médico entre el mastólogo y la mujer, teniendo en cuenta la diferencia que existe entre el concepto de cuerpo en dicha religión y en la medicina. Para una mujer católica ir al mastólogo es, quizás, parte del deber ser en términos de prevención primaria, y por ende las exigencias del examen representan cierta incomodidad, pero son asumidas con naturalidad. Es decir, que entrar al consultorio, descubrirse el torso y permitir la palpación de los senos, como acto aséptico y carente de erotismo en la técnica médica, no es vivido como acto de la ruptura de su identidad e intimidad. Sin embargo, al remitirnos al cuerpo de la mujer en el islam se debe tener en cuenta que este es pensado de manera íntima y sagrada, lo cual exige su encubrimiento, no para disimularlo ni para esconderlo, sino para mantener su sacralidad tanto en los espacios privados como en público (57).

En otras palabras, el cuerpo islámico cubierto es observante de la tradición, declara su individualidad dentro de un sistema de relaciones sociales. Los estereotipos presentados por los medios masivos de comunicación proyectan la imagen e idea que la mujer musulmana al estar cubierta, en cuanto que característica cultural, se constituye en un símbolo de sumisión y no empoderamiento. El hiyab, el velo musulmán, es pensado y representado como un objeto en contravía de la liberación femenina, que está asociada a la libertad de los derechos sexuales de las mujeres $(51,58,59)$ y a la revolución cultural que determinó la necesidad de la igualdad entre géneros, más aún si se piensa el islam como una religión profundamente patriarcal y sexista. La fotógrafa Shirin Nestat, al preguntársele por la condición de las mujeres en Irán, respondió (60): "Por supuesto, la situación en Irán ha sido muy opresiva, pero contrario a lo que la imagen es, las mujeres son extremadamente rebeldes, son muy expresivas, muy polémicas y de ninguna manera sumisas".

Claudia es una mujer colombiana convertida al islam, y desde el momento de su conversión usa hiyab, atuendo que no es obligatorio, sino que se usa en señal de devoción. Fue diagnosticada de cáncer de seno antes de su conversión; sin embargo, cuando se convirtió, ya su tratamiento estaba adelantado y tuvo que plantearse una postura que le permitiera asistir a los controles y seguir con sus cuidados. El médico tratante de Claudia 
es hombre, pero ella consideró que sería impertinente cambiar de profesional, ya que en la comunidad se le permitió mantenerlo. Además, su EPS no le ofreció ni le ofrece una médica de iguales calidades a las de su médico tratante, por ello la desesperanza se plantea como un sistema de afrontamiento. Como ella misma lo narra "[...] entonces toca dejarse atender de un hombre".

De otra parte, Claudia, con su conversión al islam y el uso del hiyab, siente que la atención médica hacia ella se ha vuelto más respetuosa, ya que la manera en que la ven es diferente; ya no es solamente una mujer más de la sociedad bogotana, ahora es una mujer cubierta en su cuerpo, y la atención médica es mucho más precavida, se le brinda más información, la antesala y los preámbulos en el consultorio son mayores, hasta que le es imposible omitir la auscultación y la solicitud de descubrir su torso para el examen. En este contexto, el trabajo del médico tratante se volvió puntual, concreto, rápido, breve y técnico. La observación, el tacto, la manipulación y el tiempo de la consulta en el cual el torso está descubierto se redujo para minimizar un acto médico que podría contradecir su fe. En el caso presentado el respeto funcionó positivamente para la persona.

En otros casos, la experiencia del examen preventivo o clínico para la detección temprana plantea retos adicionales que no solo dependen del médico, sino que involucran la cadena burocrática de atención de la mujer musulmana. La disponibilidad del sistema, el tipo de aseguramiento, el tipo de tratamiento y la actitud positiva o negativa con respecto a ellas determinan, tanto el acceso a los servicios en salud, como sus trayectorias terapéuticas sobre qué hacen, a dónde van, a quién acuden, 46 con quién hablan para definir la forma como afrontan sus necesidades en salud.

\section{Conclusiones}

Ha pasado ya más de una década desde el atentado a las torres gemelas de Nueva York en 11 de septiembre del 2001, hecho que marcó un hito en la historia de lo que llamamos Occidente y que nos remitió inmediatamente a las luchas medievales entre cristianos e infieles. Así mismo, la reciente elección de Sadiq Khan como alcalde de Londres, un ciudadano británico de padres pakistaníes, a la cabeza de un lugar icónico del mundo occidental que también sufrió un atentado con bombas por parte de $\mathrm{Al}$ Qaeda el 7 de junio de 2005. Khan puede ser considerado, en los términos de Mandami, un buen musulmán, pues defiende valores religiosos que desde la emergencia actual del terrorismo encarnan la vivencia real del islam y contradicen el estereotipo sobre esa religión.

Es claro que la sociedad británica, debido a su pasado imperial, está acostumbrada a pensar la diversidad cultural y étnica por su historia misma. No en vano países que fueron parte del imperio siguen siendo aún hoy territorios súbditos de la monarquía británica, agrupados bajo la idea de la Common Wealth, que según el juramento de coronación de la reina Isabel II, siguen un orden social "de acuerdo con sus respectivas leyes y costumbres". La Union Jack, como se le dice a la bandera británica, se ondea de alguna u otra forma en las antiguas colonias, creando una identidad a partir de rasgos particulares que hacen que Kipling, Isaiah Berlin, Salman Rushdie, Lord Beveridge, Pocahontas o Ronald Ross, entre muchos otros, habiendo nacido en el extranjero sean británicos, y que Sadiq Khan, londinense de padres pakistaníes, sea británico.

En Colombia, un país sin historias imperiales, ni grandes triunfos militares más que los hitos de la guerra de independencia, el problema del multiculturalismo ha sido un 
tema constante alrededor del nacionalismo, y es recientemente que reaparece bajo la idea de diversidad y equidad social en la agenda nacional. Jorge Bejarano escribía en El libro de la maternidad (61, p. 1):

\section{[...] que enseñe a nuestras mujeres a ser madres, no en el sentido de prepararse para aquel acto fisiológico, que lo guía la misma naturaleza, sino a hacer del fruto de sus entrañas el ejemplar auténtico de una raza o de un país que aspira a ver en sus niños la más clara manifestación de la vitalidad y cultura de sus ciudadanos.}

En esta cita es posible observar cómo la mujer, no solo por su diferencia de género, sino también por su condición de diferencia, se ubica en un lugar donde la individualidad debe estar sometida al ideal nacional.

Cuando planteamos el problema del multiculturalismo actual y su lugar en el campo de la salud, estamos interrogándonos tanto por los macrocontextos como por los microcontextos donde este concepto se escenifica. En este estudio el énfasis fue el microcontexto, donde el encuentro médico, de una parte, revela la articulación de las condiciones de vida, las culturas y las prácticas sociales con el cuidado en salud, y de otra parte, plantea la independencia de la idea de nación y estándares unificados de atención en salud con las necesidades sentidas de los grupos sociales e individuos.

En este sentido, al cuestionar icómo la diversidad cultural se integra en los microcontextos del encuentro médico?, las respuestas desde la Constitución Política parecieran evidentes (62). Sin embargo, cuando la diversidad cultural es un valor fundamental del Estado nación colombiano, la protección de esta se plantea como un derecho ciudadano, asegurando el respeto de la identidad cultural. A pesar de esto, en la práctica cotidiana del encuentro médico no se refleja, debido al dominio científico que se presume como valor superior y fundamental sobre lo cultural y lo religioso (63).

En la relación médico-paciente dentro del SGSSS los aspectos políticos, culturales, económicos, emocionales e ideológicos se constituyen en filtros que influyen en la relación entre un médico y un/una paciente musulmán. Los casos de obstetricia, ginecología y cáncer de mama muestran la complejidad del acceso a la atención, porque combinan sistemas de creencias, estilos de vida, la presentación social del cuerpo y la estructura social. Exponer un cuerpo, tocarlo, auscultarlo, observarlo, se perciben como prácticas normales dentro del encuentro médico propio de la medicina contemporánea, debido a la reificación de los cuerpos y las enfermedades.

Ahora bien, cuando la mujer musulmana objeta la intervención en su cuerpo, no solo por un tabú, porque es ignorante o por falta de educación, sino porque su cuerpo es más que un objeto de atención terapéutica y porque dentro del islam es sagrado, íntimo y se asocia a un problema de género, el acto médico dentro de la consulta es, inmediatamente, transformado. El médico, allí, deja de ser un simple proveedor en salud, para ser un intermediario entre lo íntimo, lo público y lo sagrado.

Finalmente, el encuentro médico-paciente es un lugar donde se revela la complejidad de lo que significa la diversidad poblacional del país. Detrás del conocimiento del territorio y de las necesidades planteadas a través de los sistemas de producción, de la construcción de infraestructura de saneamiento básico y de los planes de vacunación, entre muchos aspectos que fundamentan los objetivos de trabajo del Ministerio de Salud, expresados en la Política de Atención Integral en Salud, se encuentran ideales sobre estilos de vida, 
salud y control de enfermedades de la población que no necesariamente son consonantes en el marco de la vivencia de la ciudadanía. Pensar en la nación y en su cuerpo implica pensarla dentro de una unidad corporal biológica y política, pero dentro de múltiples cuerpos culturales, tal como ha sido descrito ampliamente por diversos autores a lo largo de los siglos XX y XXI.

Ser musulmán en Bogotá y ser musulmán en un encuentro médico-paciente, es develar las posibles contradicciones entre el carácter universal de aquello que es "común a todos" dentro del SGSSS y las especificidades de los diferentes grupos que constituyen la nación colombiana. Tal vez sea a partir de allí que el multiculturalismo deje de ser un enunciado legal para ser una práctica médica legitima socioculturalmente.

\section{Referencias bibliográficas}

1. Johnson JL, Bottorff JL, Browne AJ, Sukhdev G, Hilton BA, Clarke H. Othering and being othered in the context of health care services. Health Communication. 2004; 16(2):255-71.

2. Van Dijk TA. Discurso y racismo en América Latina. Barcelona: Gedisa; 2007.

3. Locke DC, Bailey DF. Increasing multicultural understanding. Thousand Oaks: Sage; 2013.

4. Irizarry Y. Selling students short: Racial differences in teachers' evaluations of high, average, and low performing students. Social Science Research. 2015; 52:522-38.

5. Pohl-Valero S. ¿Agresiones de la altura y degeneración fisiológica? La biografía del "clima" como objeto de investigación científica en Colombia durante el siglo XIX e inicios del XX. Rev Cienc Salud. 2015; 13:65-83.

6. Rica Pérez T. Minorías étnicas en El Reino Unido. Ejemplo de Sheffield. Revista Geographicalia. 1996; 33:147-65.

7. Allen DG. Knowledge, politics, culture, and gender: a discourse perspective. Canadian Journal of Nursing Research. 1996; 28(1):95-102.

8. Menéndez E. De sujetos, saberes y estructuras: introducción al enfoque relacional en el estudio de la salud colectiva. Buenos Aires: Lugar Editorial; 2009.

9. Castellanos DG. Islam en Bogotá: presencia inicial y diversidad. Bogotá: Universidad del Rosario; 2010.
10. Huntington SP. El choque de las civilizaciones y la reconfiguración del orden mundial. Buenos Aires: Paidós; 2005.

11. Said EW. Orientalismo. Madrid: Debolsillo; 2002.

12. Mamdani M. Good Muslim, bad Muslim: A political perspective on culture and terrorism. American Anthropologist. 2002; 104(3):766-75.

13. Fanon F. Piel negra mascaras blancas. Buenos Aires: Editorial Abraxas; 1973.

14. Batalla BG. La teoría del control cultural en el estudio procesos étnicos. En: Estudios sobre culturas contemporáneas. México: Universidad de Colima; 1988. p. $165-204$.

15. Fleischer S. Pasando por comadrona, midwife y médico: el itinerario terapéutico de una embarazada en Guatemala. Anthropologica. 2006; XXIV(24):51-75.

16. Duque-Páramo MC. Cultura y salud: elementos para el estudio de la diversidad y las inequidades. Investigación en Enfermería: Imagen y Desarrollo. 2007; 9(2):127-42.

17. Ferrante C, Ferreira MAV. Cuerpo, discapacidad y trayectorias sociales: dos estudios de caso comparados. Revista de Antropología Experimental. 2008; 29(8):403-28.

18. Kowal E. The politics of the gap: Indigenous Australians, liberal multiculturalism, and the end of the self-determination era. American Anthropologist. 2008; 110(3):338-48.

19. Spector RE. Cultural diversity in health and illness. J Transcultural Nursing. 2002; 13(3):197-9.

20. Koening H. Religion and medicine II: Religion, mental health and related behaviors. International Journal of Psychiatry in Medicine. 2001; 31(1):97-109.

21. Taussig M. Un gigante en convulsiones: el mundo humano como sistema nervioso en emergencia permanente. Barcelona: Gedisa; 1995.

22. Sarri K, Kafatos A. Are religions "heatlthy"? A review on religious recommendations on diet and lifestyle. Human Ecology Special Issue. 2006; 14:7-20.

23. Curlin FA. Spirituality and lifestyle: what clinicians need to know. South Med J. 2006; 99(10):1170-1.

24. Suárez R, Beltrán EM, Sánchez T. El sentido del riesgo desde la antropología médica: consonancias y disonancias con la salud pública en dos enfermedades transmisibles. Revista Antípoda. 2006; 3:123-55.

25. Cunninghame G, Robert B. Cartagena y las riberas del Sinú. Sevilla: Ediciones Espuela de Plata; 2010.

26. Vargas P, Suaza LM. Los árabes en Colombia: Del rechazo a la integración. Bogotá: Editorial Planeta; 2007.

27. Corbetta P. Metodología y técnicas de investigación social. Bogotá: McGraw-Hill; 2007.

28. Del Pino Fernando JP, Del Pino MV. La visión del otro y la conflictividad en la relación enfermerapaciente musulmán. Revista Enfermería Clínica. 2012; 22(4):191-7.

29. Taylor S, Bodgan R. Introducción a los métodos cualitativos de investigación. Barcelona: Paidós; 1992.

30. Scheper-Hughes N, Lock M. The mindful body: A prolegomenon to future work in medical anthropo- 
logy. Medical Anthropology Quarterly New Series. 1987; 1(1):6-41.

31. World Health Organization. Human papillomavirus (HPV) and cervical cancer. Disponible en: http:// www.who.int/mediacentre/factsheets/fs380/en/

32. Instituto Nacional de Cancerología. Plan decenal para el control del cáncer de Colombia, 2012-2021. Bogotá: Instituto Nacional de Cancerología; 2015.

33. República de Colombia, Ministerio de Salud. Plan Decenal de Salud Pública 2012-2021. Bogotá: Ministerio de Salud; 2013.

34. Shavers VL, Fagan P, Jones D, Klein WM, Boyington J, Moten C, Rorie E. The state of research on racial/ethnic discrimination in the receipt of health care. Am J Public Health. 2012; 102(5):953-66.

35. World Health Organization. Cribado del cáncer del cuello del útero. Disponible en: http://www.who.int/ cancer/detection/cytologyscreen/es/

36. Emerson JP. Behavior in private places: sustaining definitions of reality in gynecological examinations. Recent Sociology. 2008; 72(2):74-97.

37. Restrepo L. Médicos y comadronas o el arte de los partos: la ginecología y la obstetricia en Antioquia 1870-1930. Medellín: La Carreta; 2006.

38. Drife J. The start of life: a history of obstetrics. Postgrad Med J. 2002; 78:311-5.

39. Harding S. Ciencia y feminismo. San Sebastián de los Reyes: Morata; 1996.

40. Argüello-Avendaño HE, Mateo González A. Parteras tradicionales y parto medicalizado, ¿un conflicto del pasado? Evolución del discurso de los organismos internacionales en los últimos veinte años. LiminaR. Estudios Sociales y Humanísticos. 2014; XII(2):13-29.

41. Asociación Bogotana de Obstetricia y Ginecología (ASBog). Guía de control prenatal y factores de riesgo. Bogotá: Secretaría Distrital de Salud de Bogotá; s. f.

42. Gilmore DD. Manhood in the making: Cultural concepts of masculinity. New Haven: Yale University Press; 1990.

43. Dudink SP, Hagemann K, Clark A. Representing masculinity: Male citizenship in modern western culture. Nueva York, NY: Palgrave Macmillan; 2012.

44. Miranda M, Obregón D, Hernández M. La Organización Panamericana de la Salud y el Estado colombiano: cien años de historia; 1902-2002. Bogotá: Organización Panamericana de la Salud; 2002.

45. Ministerio de Sanidad y Política Social. Guía de práctica clínica sobre la atención al parto normal. Urdulz-Biskaia- España; 2010.

46. Famisanar. Acceso a servicios - manual de autorizaciones para IPS, 2013. Disponible en: http:// www.famisanar.com.co/mailings/famisanar_2013/ manualautorizaciones.pdf

47. Compensar EPS. El nacimiento está cerca. Disponible en: http://Compensar.Com/Salud/Documents/ Nacimientocerca.Pdf

48. Organización Panamericana de la Salud. Cáncer de mama. Disponible en: http://www.paho.org/hq/ index.php?option $=$ com_content $\&$ view $=$ category $\& \mathrm{id}=3400 \&$ layout $=$ blog \& Itemid $=3639 \&$ lang $=e s$

49. IARC. Breast Cancer Screening. IARC Handbook of Cancer Prevention. Volume 15. 2015.

50. Organización Mundial de la Salud. Cáncer de mama: prevención y control. Disponible en: http://www. who.int/topics/cancer/breastcancer/es/index3.html

51. Fundación contra el Cáncer. Cáncer de mama: una guía para pacientes - basada en la guía de práctica clínica de la Esmo V; 2013.

52. Morris D. La mujer desnuda. Madrid: Planeta; 2004.

53. Sánchez ST. Efectos psíquicos de la mastectomía (preventiva y terapéutica). Cambios en la percepción de las mujeres con cáncer de mama. Papeles del Psicólogo. 2015; 36(1):62-73.

54. Claudel PT, Hernández FS. Consecuencias psicoafectivas y sociales del cáncer de mama y de la mastectomía. Revista Costarricense de Ciencias Médicas. 1985; 6(2):29-38.

55. Powell L. Refashioning the post-mastectomy body in how to look good naked. Film, Fashion \& Consumption. 2013; 2(1):43-54.

56. López Peres MD, Lorente A, Arranz P. Imagen corporal en enfermas mastectomizadas. Psiquis: Revista de Psiquiatría, Psicología Médica y Psicosomática. 1992; 13(10):49-54.

57. Armstrong K. El islam. Bogotá: Editorial Debate; 2013.

58. Cardona-Lozada D. Mujeres y anticonceptivos, ¿liberación femenina? Perspectivas en Bioética. 2014; 18(1):12-21.

59. Cardona-Lozada D. Mujeres y anticonceptivos, ¿liberación femenina? Perspectivas en Biobioética. 2014; 18(1):12-21.

60. Neshat Shirin. Gladstone Gallery, Neshat Shirin translates the Iranian political unrest through an artistic lens. Disponible en: http://gladstonegallery.com/ sites/default/files/SN_PBS_June242015_online.pdf

61. Bejarano J. El libro de la maternidad. Bogotá: Editorial Minerva; 1924.

62. República de Colombia, Constitución Política de Colombia, 1991.

63. Suárez R, Forero AM. Itinerarios terapéuticos de los devotos del Divino Niño del 20 de Julio: entre las fisuras expertas en Salud. Bogotá: Uniandes; 2002. 